\title{
Der Hörsaal als Lernraum
}

\author{
Matthias Klatt/Tina Winter"
}

Die Rahmenbedingungen universitärer Lehre werden derzeit neu konstituiert. Aktivierende und kompetenzfördernde Lehrformate haben das Potential, die rechtswissenschaftliche Lehre erheblich zu verändern. Den Schwerpunkt der Lehrentwicklung an vielen juristischen Fakultäten bildet derzeit die Verbesserung der Lehre in den Arbeitsgemeinschaften und anderen Kleingruppenformaten. Daneben bieten sich die Umgestaltung von Vorlesungen sowie die Reformulierung von bestehenden Curricula als fachdidaktische Entwicklungsfelder an. Der vorliegende Beitrag erläutert anhand des Beispiels eines "Marktplatzes“, wie kompetenzfördernde Methoden auch in großen Vorlesungen eingesetzt werden können. Er zieht zudem Konsequenzen für generelle hochschulpolitische Strukturentscheidungen.

\section{A. Kompetenzförderung in den Rechtswissenschaften}

Die Förderung von persönlichkeitsbezogenen und berufsfeldorientierten Kompetenzen der Studierenden ist eine Kernaufgabe der Universität. ${ }^{1}$ Die überkommene universitäre Lehr- und Lernkultur ist daher in vielfacher Weise zu verändern. ${ }^{2}$ Auch die juristischen Fakultäten stehen vor dieser Herausforderung. Sie nehmen ihren Bildungs- und ihren Ausbildungsauftrag nur dann angemessen wahr, wenn sie ihre Lehre kompetenzfördernd gestalten. In dieser Hinsicht wird seit längerem ein gewisser Nachholbedarf gerade an den juristischen Fakultäten diagnostiziert. ${ }^{3}$ Dem geforderten Wandel von der Lehr- zu der Lernorientierung ${ }^{4}$ entspricht es, verstärkt aktivierende Lehrformate einzusetzen: die Studierenden müssen im Mittelpunkt der Lehrplanung stehen.

* Jun.-Prof. Dr. Matthias Klatt ist Inhaber der Juniorprofessur für Öffentliches Recht, Europarecht, Völkerrecht und Rechtsphilosophie an der Universität Hamburg. Ass. iur. Tina Winter, MoHE [Master of Higher Education], ist wissenschaftliche Mitarbeiterin an der Juniorprofessur. Die hier beschriebene Lehreinheit ist auch bei Klatt, Lehrmethoden, http:/www.matthias-klatt.de/lehre/lehrmethoden/ (01.03.2013) dokumentiert.

1 Vgl. BVerfGE 35, 79 (121, 125); aus grundrechtlicher Perspektive A.-K. Kaufhold, Die Lehrfreiheit ein verlorenes Grundrecht? (2006), S. 121 ff.; allgemeiner zur Kopplung der „Anpassung der Studienangebote an vermeintliche Bedarfe des Beschäftigungssystem[s]“ Teichler, Die Verknüpfung von Bildung und Forschung, in: Tippelt/Schmidt, Handbuch Bildungsforschung, 2010, S. 421-444 (431 ff.); mit kritischem Unterton Schenke, NVwZ 2005, S. 1000-1009 (1002).

2 Knapper Überblick über die potentiellen Handlungsfelder bei Schmidt, Zeitschrift für Pädagogik Beiheft 2005, S. 103-114.

3 Husserl, JZ 1953, S.453-456 (454); Böckenförde, FAZ 29.10.1996, S. 12; Erichsen, JURA 1998, S. 449-453 (450); Hesse, JZ 2002, S. 704-708 (706); Kahlo, Wozu heute Rechtswissenschaft lehren und studieren?, in: Graul, Gedächtnisschrift für Dieter Meurer, 2002, S. 583-621 (599); Hoffmann-Riem, JZ 2007, S. 645-652 (647 ff.).

4 „The shift from teaching to learning “ geht als Wortschöpfung wohl zurück auf UNESCO, A Shift From Teaching To Learning, http://portal.unesco.org/education/en/ev.php-URL_ID=26923\&URL_DO=DO _TOPIC\&URL_SECTION=201.html (01.03.2013); zum klassischen Streit in der bildungstheoretischen Forschung siehe Reusser, Unterricht zwischen Wissensvermittlung und Lernen lernen, in: Finkbeiner, Lehren und Lernen im Kontext empirischer Forschung und Fachdidaktik, 2001, S. 106-140. 


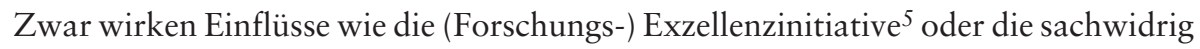
einseitige Forschungsorientierung in der etablierten Berufungspraxis ${ }^{6}$ einer Verbesserung der Lehrqualität häufig entgegen. Jedoch sind in den letzten Jahren Initiativen mit dem gemeinsamen Ziel entstanden, die Qualität der Lehre an den juristischen Fakultäten voranzubringen. Dazu gehören die Bemühungen um die Etablierung einer rechtswissenschaftlichen Fachdidaktik ${ }^{7}$ ebenso wie eine Reihe von Tagungen ${ }^{8}$ oder der kritische Bericht der umfassender angelegten Arbeitsgruppe „Perspektiven der Rechtswissenschaft" des Wissenschaftsrates. ${ }^{9}$ Letztlich entscheidend für den Erfolg dieser Bemühungen sind aber weder die Schaffung großer zusätzlicher Strukturen noch eine Komplettreform der Ausbildungsgesetze. Entscheidend ist vielmehr das, was in den Lernräumen der Fakultät, also im Hörsaal und im Seminarraum, konkret geschieht und Lehrende und Studierende unmittelbar betrifft. Unsere These lautet, dass im Sinne einer „Reform der kleinen Schritte“ (bottom-up) viele Handlungsmöglichkeiten bestehen, die mit vergleichsweise geringem Aufwand hohe Wirkung erzielen. Eine dieser Möglichkeiten wollen wir hier näher erläutern.

Kompetenzförderung darf dabei nicht als wissenschaftsferne Berufsvorbereitung missverstanden werden. Vielmehr basiert sie auf einem anspruchsvollen Kompetenzbegriff. ${ }^{10}$ Dieser umfasst neben der fachlichen Kompetenz im engeren Sinne auch die

5 Besonders deutlich ist die Analyse von Kielmansegg, FAZ 2012 (8.8.2012), S. N5.

6 Vgl. Klatt/Koller, Forschung und Lehre 19 (2012), S. 448-449; Die Junge Akademie, Zur Zukunft der Lehre an Universitäten in Deutschland, http://www.diejungeakademie.de/fileadmin/user_upload/Lite ratur/pdf/Positionspapier_Lehre.pdf (01.03.2013), S. 9, 20; Die Junge Akademie, Qualität statt Quantität - auch in Berufungsverfahren, http://www.diejungeakademie.de/fileadmin/user_upload/Lit eratur/pdf/Positionspapier_QualitaetstattQuantitaet.pdf (01.03.2013), S. 2 f.

7 Brockmann/Dietrich/Pilniok, JURA 31 (2009), S. 579-585. Siehe. das „Zentrum für rechtswissenschaftliche Fachdidaktik (ZerF)“ an der Universität Hamburg, das „Zentrum für juristisches Lernen“ an der Bucerius Law School, das Projekt „Recht Aktiv“ an der Universität zu Köln, das „Institut für Rechtsdidaktik “ an der Universität Passau sowie das Projekt REGINA an der Universität Regensburg.

8 Siehe die Tagung der Hochschulrektorenkonferenz „Juristenausbildung heute: Impulse für Studium und Lehre" vom 13./14. November 2012 in Bonn, Tagungsdokumentation: http://www.hrk-nexus.d e/aktuelles/tagungsdokumentation/jura-tagung/ (01.03.2013), sowie die Sammelbände Hof/ Olenhusen, Rechtsgestaltung - Rechtskritik - Konkurrenz von Rechtsordnungen (2012); Griebell Gröblinghoff, Von der juristischen Lehre (2012); Brockmann/Brockmann-Dietrich-Pilniok, Methoden des Lernens in der Rechtswissenschaft (2012); Brockmann/Dietrich/Pilniok, Exzellente Lehre im juristischen Studium (2011).

9 Perspektiven der Rechtswissenschaft in Deutschland. Situation, Analysen, Empfehlungen

(Drs. 2558-12), November 2012, http://www.wissenschaftsrat.de/index.php?area=\&ptyp=\&year $=20$ $12 \&$ keyword=Rechtswissenschaft $\&$ suchen $=$ suchen $\& \mathrm{id}=836 \& \mathrm{rpp}=\&$ search $\mathrm{data}=1 \& \mathrm{~L}=0$ (01.03.2013), S. $53 \mathrm{ff}$.

10 Überblick bei Klieme/Hartig, Zeitschrift für Erziehungswissenschaft 2008, S. 11-29; Weinert, Concept of Competence, in: Rychen/Salganik, Defining and Selecting Key Competencies, 2001, S. 45-65. Zur handlungsorientierten Ausrichtung des Kompetenzbegriffs M. Kaufhold, Kompetenz und Kompetenzerfassung 2006 (22 ff.). Zur Kompetenz als „Lernerfolg im Hinblick auf die Person des Lernenden“ Raithel/Dollinger/Hörmann, Einführung Pädagogik, 3. Aufl. (2009), S. 39. Zum europäischen Kontext Bolder, Arbeit, Qualifikation und Kompetenz, in: Tippelt/Schmidt, Handbuch Bildungsforschung, 2010, S. 813-843 (821 ff.). „Learning Outcomes als Kompetenzen“ beschreibt Wildt, Praxisbezug revisited - Zur hochschuldidaktischen Rekonstruktion von Theorie-Praxis-Verhältnissen in Studium und Lehre, in: Merkt/Mayrberger, Die Qualität akademischer Lehre, 2007, S. 59-72 (66). Kritisch zum 
Bildung von Persönlichkeiten, ${ }^{11}$ die über den fachlichen Horizont hinausschauen, über soziale und kommunikative Fähigkeiten ${ }^{12}$ verfügen und sich ihrer ,gesellschaftsgestaltenden Verantwortung " 13 bewusst sind. Diese Lehrziele erfordern die Entwicklung fachdidaktischer Konzepte, die auch den Freiraum zur Reflexion über die eigenen Methoden und Grenzen einschließen. Die juristische Lehrkultur ist allerdings nach wie vor auf eine allzu passive Wissensrezeption durch die Studierenden ausgerichtet. Lehr- und Lernkonzepte der juristischen Fakultät sind daher neu zu gestalten. Diese Neugestaltung lässt sich für das Lernen in kleinen Gruppen noch relativ leicht vornehmen. Für Veranstaltungen mit vielen Studierenden jedoch, beispielsweise für Vorlesungen, wird dies zu Recht als schwieriger und aufwändiger angesehen. ${ }^{14}$ Prinzipiell ist das traditionelle Konzept der Vorlesung - der Lehrende als Vortragender und die Studierenden als seine Zuhörerschaft - nur bedingt geeignet, Studierende aktiv zu beteiligen. Der Vorteil des Hörsaals indes ist seine räumliche Größe. Nutzt man diese, wird aktivierende Lehre auch in großen Vorlesungen möglich.

Bekannte Konferenzformate wie Markt der Möglichkeiten, ${ }^{15}$ poster walk oder open space $^{16}$ sprechen die Kompetenzen der Studierenden auch in großen Gruppen und direkt im Hörsaal an. Die aktive Einbindung der Studierenden in die Vorbereitung dieser Settings ist ein Bestandteil des didaktischen Konzepts, als das sich diese Konferenzformate interpretieren lassen. Ebenso gehört es zu diesem Konzept, den Hörsaal als Lernraum zu erschließen. Weil diese Formate auf wissenschaftlichen Konferenzen zum Einsatz gelangen, ${ }^{17}$ ist dieser didaktische Ansatz besonders wissen-

Grundkonzept Klein/Dungs Susanne, Einleitende Bemerkungen zur Standardisierung der Bildung, in: Klein/Dungs, Standardisierung der Bildung auf dem Prüfstand, 2009, S. 7-22 (10); Siehe zudem Bundesministerium für Bildung und Forschung/ Kultusministerkonferenz, Deutscher Qualifikationsrahmen, http://www.deutscherqualifikationsrahmen.de/ (01.03.2013).

11 Vgl. hierzu auch Böckenförde, Vom Ethos der Juristen (2010), S. 35; Wolf, Fragwürdigkeit und Notwendigkeit der Rechtswissenschaft (1953), S. 9; Lenzen, Die Zeit 15.3.2012, S. 77 (77).

12 Vgl. Voßkuble, Rechtswissenschaft 2010, S. 326-346 (345).

13 Rüthers, JuS 51 (2011), S. 865-870 (865).

14 Vgl. Dauner-Lieb/Wessel/Pernice-Warnke, Das Projekt "Recht Aktiv" - Ein ganzheitliches Konzept für exzellente Lehre im juristischen Studium, in: Brockmann/Dietrich/Pilniok, Exzellente Lehre im juristischen Studium, 2011, S. 185 (196).

15 Siehe Schettler, Methoden, http://www.lis.bremen.de/sixcms/media.php/13/Schettler_Methoden_200 9.pdf (01.03.2013).

16 Vgl. Owen, Open space technology (2008); Reich, Open Space, http://www.uni-koeln.de/hf/konstru $\mathrm{kt} /$ didaktik/openspace/frameset_open.html (27.2.2013).

17 Vgl. z.B. für die Rechtswissenschaften die Konferenz „Juristenausbildung heute: Impulse für Studium und Lehre“ am 13./14. November 2012, Hochschulrektorenkonferenz, Jura - HRK nexus, http://ww w.hrk-nexus.de/faecher/rechts-wirtschafts-und-sozialwissenschaften/jura/ (27.2.2013). 
schaftsnah. Die dringend zu reanimierende Einheit ${ }^{18}$ von Forschung und Lehre wird so zu einer im Hörsaal gelebten Wirklichkeit.

Ein volles Curriculum sorgt dafür, dass Studierende der Rechtswissenschaften immer seltener an forschungsnahen Lehrangeboten wie Seminaren partizipieren. ${ }^{19}$ Es ist wichtig, in der Ausbildung „mehr Raum für Wissenschaft“ ${ }^{20} \mathrm{zu}$ schaffen. Formate wie der „Marktplatz“ ermöglichen es, die Studierenden in die Kommunikationskultur der Wissenschaft einzuführen. Es schafft in Vorlesungen Raum für Abwechslung, Individualität und inhaltlichen Austausch. Aus einer an Lernzielen orientierten Perspektive erhalten Studierende die Möglichkeit, sich beispielsweise über die Gestaltung von Postern mit rechtswissenschaftlichen Themen auseinanderzusetzen. Den Studierenden bietet sich die Freiheit, „ihre Wege selbst zu suchen“, ${ }^{21}$ um sich das fachliche Wissen zu erschließen. Das „Erfolgserlebnis eigener Kreativität und selbständigen Denkens“ 22 das damit unmittelbar verbunden ist, kann als eine positive Lernmotivation nicht hoch genug geschätzt werden. Im Folgenden beschreiben wir die exemplarische Umsetzung dieses Ansatzes am Beispiel des „Marktplatzes Rechtsphilosophie“, der in einer zweistündigen Vorlesung Rechtsphilosophie im Sommersemester 2012 stattgefunden hat.

\section{B. „Marktplatz Rechtsphilosophie“}

Warum gilt Recht? Welcher Zusammenhang besteht zwischen Recht und Moral? Muss unmoralisches Recht befolgt werden? Diese Fragen stellen Kernthemen der Rechtsphilosophie dar. Sie wurden zunächst in der Vorlesung Rechtsphilosophie im Sommersemester 2012 in mehreren Stunden in klassischer Weise behandelt. Zur Vertiefung wurde der Hörsaal in einer weiteren Vorlesungsstunde in einen „Marktplatz“ verwandelt. Dafür hatten 15 Studierende als lead learner allein oder zu zweit Poster im Format DIN A0 zu verschiedenen Unteraspekten des Themas „Recht und Moral“ vorbereitet. Die Themen reichten vom staatlichen Tötungs- und Folterverbot, dem Umgang mit NS-Kriegsverbrechern und der Bestrafung von ehemaligen Grenzsoldaten der DDR bis hin zu einzelnen Rechtsphilosophen (Aristoteles, Sokra-

18 Vgl. Klatt/Koller, Forschung und Lehre 19 (2012), S. 448-449 (448); Stolleis, NJW 2001, S. 200-202 (201); Möllers/Voßkuble, Die Verwaltung 36 (2003), S. 321-332 (321); Schmidt-Aßmann, JZ 1995, S. 2-10 (2 ff.). Zu dem Ansatz, die Erstellung typischer juristischer Publikationsformate wie Urteilszusammenfassungen und -analysen gezielt in die Lehre einzubinden und Praxis und Wissenschaft ganz generell frühzeitig im Grundstudium zu verzahnen Dauner-Lieb/Wessel/Pernice-Warnke, Das Projekt „Recht Aktiv“ - Ein ganzheitliches Konzept für exzellente Lehre im juristischen Studium, in: Brockmann/Dietrich/Pilniok, Exzellente Lehre im juristischen Studium, 2011, S. 185-203 (192); DaunerLieb, Lehrforschung und forschendes Lernen im Jurastudium, in: Hof/Olenhusen, Rechtsgestaltung Rechtskritik - Konkurrenz von Rechtsordnungen, 2012, S. 138-151 (149).

19 Möllers/Voßkuble, Die Verwaltung 36 (2003), S. 321-332 (327); Heinig/Möllers, FAZ 21.4.2011, S. 7.

20 Heinig/Möllers, FAZ 21.4.2011, S. 7.

21 Hesse, JZ 2002, S. 704-708 (706).

22 Rüthers, JuS 51 (2011), S. 865-870 (865). 
tes, Kant, Radbruch, Kelsen). Während des Marktplatzes präsentierten die Studierenden ihren Kommilitonen zunächst 60 Minuten lang ihre Poster. Sie diskutierten miteinander über das Verhältnis von Recht und Moral. Eine Podiumsdiskussion unter Beteiligung aller lead learner schloss die Veranstaltung ab. Dort schilderten die Studierenden ihre fachlichen Erkenntnisse und Eindrücke, die sie bei der Erarbeitung ihrer Poster gewonnen hatten, und beantworteten Fragen aus dem Plenum. Das Gelingen solcher auf Interaktion und Kommunikation ausgerichteten Lehrkonzepte setzt ein klares Design ebenso voraus wie dessen sorgfältige Umsetzung.

\section{Didaktisches Konzept}

Unser Konzept des „Marktplatzes“ verknüpfte verschiedene hochschuldidaktische Ideen. Prägend für die Veranstaltung waren das lead learner-Konzept, die Konferenzmethoden des „Marktes der Möglichkeiten“ und des poster walk sowie der aktivierenden Methode der Podiumsdiskussion. Ziel war die Gestaltung einer Lerneinheit, die einerseits eine inhaltliche Vertiefung der zuvor behandelten rechtsphilosophischen Themen zulässt. Andererseits sollten die Studierenden die Möglichkeit erhalten, den Facettenreichtum des Themas „Recht und Moral“ sowie dessen praktische Relevanz in der gemeinsamen Diskussion zu erörtern. Die Vorlesungsstunde sollte so organisiert sein, dass sich die Studierenden Fachwissen eigenständig aneignen.

Der „Markt der Möglichkeiten“ ist eine Konferenzmethode, die kreative Denkprozesse unterstützt. Verschiedene Gruppenarbeitsergebnisse werden den Marktbesuchern als Poster an einem Marktstand präsentiert und diskutiert. Im lead learnerKonzept nehmen einige Studierende aus einer großen Gruppe während einer Vorlesung eine leitende Rolle ein, indem sie z.B. Fragen ihrer Kommilitonen an den Lehrenden weiterleiten. ${ }^{23}$ Dadurch soll die Hemmung vieler Studierenden, Fragen zu stellen, abgebaut und eine Gesprächskultur aufgebaut werden. Als Aussteller auf dem „Marktplatz Rechtsphilosophie“ sollten Studierende gewonnen werden, die bereit sind, sich intensiv mit einem speziellen Thema auseinanderzusetzen und dazu ein entsprechendes Poster anzufertigen. Auch die Podiumsdiskussion ist eine Methode, die in Großveranstaltungen eingesetzt werden kann, um Studierende aktiv einzubeziehen. ${ }^{24}$ Neben der Vertiefung des rechtsphilosophischen Fachwissens waren die Förderung von Diskussions- und Präsentationskompetenz wesentliche Lernziele.

\section{Vorbereitung}

Die Veranstaltung fand in der Mitte des Semesters statt. Die Vorbereitung begann jedoch bereits in der ersten Vorlesungsstunde. Wir haben drei Wochen lang mit Fly-

23 Möhrle, ZfB 1994, S. 41-52 (41).

24 Hufer, Methodenlexikon: Podiumsdiskussion (sowi-online), http://www.sowi-online.de/praxis/metho de/podiumsdiskussion.html (01.03.2013). 
ern in der Vorlesung und über Facebook für diese Sonderveranstaltung geworben. Ziel war es, Neugierde zu wecken und die Vorteile der Lehrmethode zu kommunizieren. Die Themen waren von uns vorgegeben, konnten aber auch frei gewählt werden, soweit sie zum Themenkreis gehörten.

Interessierte Studierende konnten sich zunächst unverbindlich anmelden. Mit diesem kleinen Kreis wurde im Anschluss an die dritte Vorlesungsstunde eine Informationsveranstaltung durchgeführt. Wir sprachen mit den Interessenten über die Anforderungen, Erwartungen und die jeweiligen fachlichen Interessen. Dabei haben wir den Studierenden auch Unterstützung während der Vorbereitung der Poster zugesagt. Im Anschluss konnten sich die Studierenden entweder zu Teams zusammenfinden oder entscheiden, eines der Themen allein zu bearbeiten. Die meisten Studierenden entschieden sich für die erste Variante. Insgesamt wurden Themen für neun Poster vergeben. Dabei waren Studierende vom ersten bis zum achten Fachsemester sowie ein Kontaktstudent vertreten.

Die Gliederung der anschließenden Arbeitsphase orientierte sich an dem Prozess, in dem wissenschaftliche Poster idealtypisch entstehen. ${ }^{25}$ Wir haben den Studierenden zunächst eine kleine Literaturliste ausgehändigt. Sie enthielt einen allgemeinen Aufsatz zum Oberthema „Recht und Moral“, der knapp und präzise eine einheitliche Terminologie einführte, die auch bereits aus der Vorlesung bekannt war. ${ }^{26}$ Außerdem gab es zu den spezifischen Themen jeweils einige Literaturempfehlungen. Die Studierenden wurden ermutigt, selbst nach Literatur zu recherchieren oder mit Nachfragen zu passenden Quellen auf uns zuzukommen. Nach der Hälfte der Vorbereitungszeit, die insgesamt 14 Tage umfasste, reichten die Studierenden einen Stichwortzettel mit der beabsichtigen Gliederung für das Poster sowie den von ihnen ausgewählten Inhalten ein. Zu diesem Stichwortzettel erhielten die Studierenden von uns Feedback, und es konnten gegebenenfalls Einzelheiten für das Poster besprochen werden. Anschließend verblieben den Studierenden noch knapp zwei Wochen, um das Poster anzufertigen. Hierfür wurde ihnen ein Style Guide zur Verfügung gestellt, in dem wir die für großformatige Poster relevanten didaktischen und visuellen Gestaltungsprinzipien zusammengestellt hatten. Zwei Tage vor der Veranstaltung wurden die Poster für die Studierenden im Rechenzentrum der Universität im Format A0 ausgedruckt.

\section{Durchführung}

Im Vorfeld der Veranstaltung wurden alle Teilnehmer der Vorlesung nochmals auf die Besonderheit des Termins aufmerksam gemacht. Wir haben auch eine kleine An-

25 Ruhl, Das Poster in der Wissenschaft (2011); Christian-Albrechts-Universität Kiel, Hinweise zur Gestaltung von Postern, http://www.rz.uni-kiel.de/ausgabe/postermemo/ (27.2.2013).

26 Es handelte sich um Alexy, Marburger Jahrbuch Theologie 14 (2002), S. 83-92. 
kündigung auf der Website der Fakultät platziert, um interessierten Studierenden, die nicht regelmäßig die Vorlesung besuchten, die Teilnahme zu ermöglichen. Von einer größeren Werbung haben wir aber abgesehen, um die geschützte Lernatmosphäre zu erhalten.

Die Poster wurden im Hörsaal verteilt aufgehängt. Sobald die „Standbetreuer“ vor Ort an ihrem Poster standen, begann der Marktplatz ohne weitere Einführung. Alle Besucher des Marktes konnten 60 Minuten lang an den Marktständen die Inhalte der Poster mit den Experten diskutieren. Um die Lektüre der Poster und die Diskussionen der Studierenden untereinander anzuregen, wurde allen Besuchern zuvor ein Blatt mit Fragen zu den einzelnen Postern ausgehändigt. Neben jedem Poster hing zudem eine Flipchartfolie, auf die jeder Besucher Kommentare oder Fragen schreiben konnte. Mit der Zeit entstand dadurch neben den Gesprächen auch eine schriftliche Diskussion. Zum Abschluss und als Ergebnissicherung versammelten sich die Experten für 30 Minuten zu einer Podiumsdiskussion. Nach einer knappen Zusammenfassung der einzelnen Themen gaben die Studierenden ihre eigenen Erkenntnisse zu dem von ihnen bearbeiteten Thema wieder. Dabei wurden dann auch alle Besucher des Marktes einbezogen: Kommilitonen konnten Fragen stellen und Einzelaspekte diskutieren.

\section{Nachbereitung}

Die Nachbereitung der Lehreinheit begann noch im Hörsaal. Allen Studierenden wurden Feedbackbögen in Form von Minute Papers ausgeteilt. ${ }^{27}$ Darin konnten sie ihre Einschätzung zu Ablauf und Inhalt sowie zum eigenen Lernertrag notieren. Auch nach Verbesserungsvorschlägen für eine Wiederholung dieser Lerneinheit wurde gezielt gefragt. Die Expertengruppe wurde anschließend zu „Wine \& Strawberries“ eingeladen, um ihnen Anerkennung auszusprechen und über den Verlauf des Lehrprojektes zu diskutieren. Zudem bestand für diese Gruppe ebenfalls die Möglichkeit, ihre Anmerkungen in einem Minute Paper zusammenzufassen.

\section{Reflektion der Lehreinheit}

Rückblickend ist die Lehreinheit unserer Einschätzung nach gelungen. Für uns war die Entwicklung und Durchführung dieses aktivierenden Lehrformats bereichernd. Dennoch zeigten sich Schwierigkeiten und Grenzen des gestalterischen Ansatzes. Die folgende Reflektion berücksichtigt unsere eigenen Eindrücke ebenso wie das schriftliche Feedback der Studierenden.

27 Harwood, Journal of Chemical Education 73 (1996), S. 229; Reich, Blitzlicht, http://methodenpool.u ni-koeln.de/download/blitzlicht.pdf (01.03.2013). 


\section{Innovative Gestaltung von Großvorlesungen}

Die Bereicherung klassischer rechtswissenschaftlicher Lehre mit studierendenzentrierten Lehrformaten stellt nicht nur im Hörsaal eine Herausforderung dar. Gerade im Hörsaal aber wird besonders deutlich, wie stark neue Methoden das Ausgangskonzept einer Lehrveranstaltung verändern können. Für die innovative Gestaltung von Großvorlesungen bestehen viele Möglichkeiten, die sich im Wesentlichen in zwei Kategorien einteilen lassen. Die Einführung neuer didaktischer Elemente kann sich einerseits darauf beschränken, Studierende zur Auflockerung zu einzelnen Fragen ins Gespräch zu bringen, wie das zum Beispiel in Buzz Groups geschieht. ${ }^{28}$ Solche kleineren Methoden können ohne Vorbereitung auch spontan eingesetzt werden. Demgegenüber wirken sich größere Gestaltungsansätze wie projektorientiertes, handlungsorientiertes, problembasiertes oder forschendes Lernen auf die Gestaltung der gesamten Lehreinheit aus. Denn ihre Vorstellung von Lehre und Lernen unterscheidet sich noch wesentlich stärker von der gängigen rechtswissenschaftlichen Lehre. ${ }^{29}$

Bei der Auswahl der Lehrmethoden sollte vor allem deren Eignung, die Kompetenzentwicklung der Studierenden zu fördern, berücksichtigt werden. Dies stand auch bei dem „Marktplatz Rechtsphilosophie“ im Vordergrund. Jedenfalls diejenigen Studierenden, die sich mit eigenen Postern am Marktplatz beteiligten, konnten für ein überschaubares Thema ihre Recherchekompetenz ebenso entwickeln wie die Kompetenz, das erarbeitete Fachwissen auf das Wesentliche zu fokussieren und auf dem Poster darzustellen. Sie konnten ihr Poster den Kommilitonen präsentieren und die fachlichen Inhalte erklären und verteidigen. Die herausgehobene Rolle, die Studierende in einem solchen Lernsetting wahrnehmen, führt nicht nur dazu, dass ihnen seitens ihrer Kommilitonen Anerkennung zuteil wird. Vielmehr wirkt sie zugleich als Motivation, sich auch in anderen Lehrveranstaltungen aktiver zu beteiligen. Die „Besucher“ des Marktplatzes wiederum konnten sich interessegeleitet zwischen den Marktständen bewegen, also anders als üblich unabhängig von einer Stoffauswahl des Lehrenden zwischen den Inhalten eigenständig auswählen und individuelle Schwerpunkte setzen. ${ }^{30}$ Nachfragen, Zuhören und Diskutieren konnten sie außerdem trainieren. Die Entwicklung der fachlichen Inhalte wurde bei den Besuchern durch die ausgeteilten Fragen stimuliert. Insgesamt bot sich den „Besuchern“ aufgrund der Vorarbeit der lead learner die Möglichkeit, innerhalb sehr kurzer Zeit einen sehr breiten Überblick über das Generalthema zu erhalten, der in der ausge-

28 Schiefner, Buzzgroups, http://www.hochschuldidaktik.uzh.ch/hochschuldidaktikaz/Vorlage_A-Z_Bu zzgroups.pdf (01.03.2013). Überblick über aktivierende Methoden bei Peterssen, Kleines MethodenLexikon, 2. Aufl. (2001); Macke/Hanke/Viehmann, Hochschuldidaktik (2008).

29 Überblick bei Tippelt, Vom projektorientierten zum problembasierten und situierten Lernen - Neues von der Hochschuldidaktik?, in: Reiber, Entwicklungslinien der Hochschuldidaktik, 2007, S. 137155.

30 Zur Bedeutung der Neugier und des eigenen Interesses für einen erfolgreichen Lernprozess Bruner, Harvard Educational Review 31 (1961), S. 21-32 (22). 
stellten Vielfalt nur schwer innerhalb einer 90-minütigen Vorlesung zu erreichen gewesen wäre.

Das Potential der „Marktplatz-Methode“ liegt wie bei vielen Lehrformen, die die Arbeit an kleinen Projekten integrieren, zudem darin, dass die Lernprozesse der Studierenden deutlich forschungsnäher ausgestaltet werden können. ${ }^{31}$ Es werden forschungsähnliche Handlungsweisen eingeübt. Über die Auswahl der Themen und der Literaturempfehlungen kann die Forschungsnähe der Methode zusätzlich betont werden.

\section{Vorbereitung}

Der Aufwand der Lehrenden für die Vorbereitung verlagert sich vom Inhalt zu der Aufgabe, die Rahmenbedingungen für die Lernprozesse der Studierenden zu garantieren. Eine Entlastung ist damit freilich nicht verbunden. Denn ein einigermaßen fester Rahmen an klaren, auch zeitlichen Vorgaben, ergänzt um Informationstreffen, Feedback zum Stichwortzettel und Klärung von Rückfragen hat sich als hilfreich erwiesen.

Die Hemmschwelle, sich auf die Entwicklung solcher neuer Konzepte als Alternative zur herkömmlichen Vorlesungsgestaltung einzulassen, wird in aller Regel hoch sein. Der Aufwand ist jedenfalls bei einer erstmaligen Konzeptentwicklung und -umsetzung beträchtlich. Zwar stellt die Betreuung der lead learner im Vergleich zu herkömmlichen Vorlesungen einen Zusatzaufwand dar. Wenn man jedoch mit diesem Konzept die Verantwortung für das Gelingen der Lehrveranstaltung auch stärker in die Hände der Studierenden legt, ist es erforderlich, diese entsprechend zu betreuen und in die Lehrveranstaltungsentwicklung einzubeziehen. Abgesehen davon ist der Vorbereitungsaufwand dem einer Vorlesungseinheit nicht unähnlich. Lediglich die Schwerpunktverschiebung vom Inhalt zur Struktur sowie die Neuartigkeit einzelner Planungs- und Vorbereitungsschritte lassen den Eindruck höheren Aufwands entstehen. Bereits bei einer zweiten Durchführung sollte der Aufwand erheblich geringer erscheinen, weil auf Erfahrungswissen zurückgegriffen werden kann.

Die lead learner betonten in ihrem Feedback, dass die zur Vorbereitung der Post zur Verfügung stehende Zeit (insgesamt 4 Wochen) jedenfalls ausreichend gewesen sei. Teilweise wünschten sie sich entweder ein bisschen mehr Zeit oder eine intensivere Betreuung in der Vorbereitungsphase. Es wurde angeregt, dass sich die Gruppe auch in der Vorbereitungsphase nochmals trifft, um die Inhalte und den Stand der Poster zu diskutieren oder eine bessere Vorbereitung auf die fachlichen Diskussionen zu

31 Insbesondere in kleineren Studiengängen aber auch stark abhängig von den einzelnen Lehrpersonen ist dieser Trend zu beobachten, vgl. dazu nur die zahlreichen Praxisbeispiele in Klatt/Koller, Lehre als Abenteuer (2012). 
ermöglichen, die sie während der Veranstaltung mit ihrem Kommilitonen führen sollen.

\section{Durchführung}

Bei der Durchführung ist es wichtig, sich strikt an die Sequenzierung der Lehrveranstaltung zu halten (Einführung, Hauptteil, Ergebnissicherung, Feedback). Die Studierenden sollten über den zeitlichen Ablauf informiert werden. Beides sorgt dafür, dass die Veranstaltung nicht in Strukturlosigkeit abgleitet. Es hilft den Studierenden, das Lehrformat zu akzeptieren und für sich zu nutzen.

Im Feedback wurde angeregt, den Beginn des Marktplatzes anders zu gestalten. Hier könnte noch eine weitere inhaltliche Einführung zur Orientierung geboten werden. Vorgeschlagen wurde auch, den Marktplatz mit einer kurzen Vorstellung der einzelnen Poster-Themen durch die jeweiligen Experten zu beginnen. Damit würde freilich auch der Zeitbedarf für die Veranstaltung insgesamt erhöht, der normale Vorlesungsrahmen mithin gesprengt. Von hohem Interesse ist aber jedenfalls die Rückmeldung, dass Studierende offenbar stärker bereit sind, Zeit in Lehrveranstaltungen zu investieren, wenn sie den didaktischen Mehrwert verstanden haben. Weiter wurde vorgeschlagen, mittels einer Glocke abgeschlossene Sequenzen zu schaffen und das Wechseln der Besucher zum nächsten Marktstand zu strukturieren. Auf diese Weise könnte verhindert werden, dass Besucher am neuen Poster auf eine bereits laufende Diskussion stoßen, deren Beginn sie nicht mitbekommen haben. Einige Rückmeldungen der Studierenden seien beispielhaft genannt:

- „Ich finde den heutigen Marktplatz ein wunderbares Beispiel in Richtung interessengeleitetes Studium: Die Studenten wurden motiviert, sich mit einem Thema ihrer Wahl näher zu beschäftigen und sich Meinungen zu bilden, die sie in familiärer Atmosphäre verstehen dürfen. Diese Initiativen müssen im Sinne eines Wandels der Atmosphäre an der rechtswissenschaftlichen Fakultät gestärkt werden. “

- „Es war sehr angenehm, alles in dem jeweils eigenen Tempo abhandeln zu können. Zudem war es lehrreich und erfrischend, zu jedem Thema eine Diskussion führen zu können."

- „Sehr gut für Kontakt mit Kommilitonen/innen - Verbindung schaffend - Zusammengehörigkeit“

- „Ich fand den Marktplatz von heute eine sehr gelungene Abwechslung zur generellen Vorlesung und bin der Meinung, dass man so was viel öfter machen sollte, da man sich in diesen (knapp) zwei Stunden viel intensiver mit dem Stoff und vor allem seinen eigenen Gedanken befasst.

- "Jeder konnte individuell entscheiden, was er oder sie sich anhören wollte (viele Interessenfelder abgedeckt); dadurch wurde auch ein höherer Lernerfolg erzielt“

- „Alle Experten waren gut vorbereitet und konnten Fragen kompetent beantworten “ 
- „Mir hat der Marktplatz sehr gut gefallen! Ich bin sehr von dem Modell überzeugt, über Gespräche und Problemstellungen Fragen und Inhalte zu verstehen.“

- „In kleinen Gruppen kommt fast jeder zu Wort. So kann man Fragen stellen und über Dinge nachdenken, mit denen man sich sonst selten beschäftigt.

Der Marktplatz war durch die vorangegangen Vorlesungsstunden zum Thema Recht und Moral inhaltlich sehr gut vorbereitet, was der Qualität der Poster und Diskussionen sichtlich zugutekam. Freilich war die Einbettung des Marktplatzes in die Gesamtvorlesung so beschaffen, dass der Marktplatz als Vertiefungsveranstaltung das Modul „Recht und Moral“ abschloss. In den folgenden Veranstaltungen ging es mit rechtstheoretischen Themen weiter. Dadurch ergaben sich keine direkten Möglichkeiten, den Marktplatz noch einmal aufzugreifen und an die dort diskutierten Themen anzuschließen.

Wünschenswert wäre es, den Studierenden über das Erstellen und Präsentieren der Poster auch den Scheinerwerb zu ermöglichen. Derzeit müssen alle Studierenden der Veranstaltung eine Abschlussklausur schreiben, um den Grundlagenschein zu erwerben. Dafür müsste aber im Fach Rechtswissenschaften deutlich mehr Wissen über kompetenzorientierte Prüfungsformate aufgebaut werden. Hier zeigt sich, dass innovative Lehrgestaltung auch mit einer Erprobung neuer Prüfungsformate einhergehen sollte.

\section{Transfermöglichkeiten und Entwicklungsperspektiven}

Unsere Erfahrungen mit der Lehreinheit und die Rückmeldung der Studierenden legen es nahe, solche großen Gestaltungsszenarien wie den "Marktplatz“ für die rechtswissenschaftliche Lehre zu erschließen. Hierbei muss jedoch auf das rechte Maß geachtet werden, damit Lehrende und Studierende bei Vorbereitung und Durchführung nicht überlastet werden.

Ein punktueller Einsatz erscheint sehr gut geeignet, einzelne Lehreinheiten einer Vorlesung aufzulockern. Der Ansatz, studentische Arbeitsergebnisse in Form von Postern zu präsentieren, ist auf jede rechtswissenschaftliche Lehrveranstaltung mit beliebigem Inhalt übertragbar. Vor allem Großveranstaltungen, die in der Regel eine niedrige Partizipationsrate im Sinne aktiver Beteiligung aufweisen, können davon profitieren. Für die Lehrenden bietet sich zudem die nicht zu unterschätzende Gelegenheit, vertiefte Einblicke in die Arbeitsweise und in das Denken der Studierenden zu erhalten. Diese Einblicke sind sonst in Vorlesungen kaum möglich, für die erfolgreiche Gestaltung studentischer Lernprozesse aber unentbehrlich.

Darüber hinaus stellen Poster ein Format für Prüfungsleistungen dar, das nicht nur in Vorlesungen, sondern beispielsweise auch in Seminaren verwendet werden könnte. Eine besonders innovative Fortentwicklung wäre es dabei, wenn in solchen Lehrver- 
anstaltungen auch der Nachweis über sogenannte Schlüsselqualifikationen erbracht werden könnte. Auf diese Weise würden die im engeren Sinne fachbezogene Lehre und die Vermittlung von Schlüsselqualifikationen sinnvoll integriert, anstatt wie bisher in getrennten Lehrveranstaltungen künstlich separiert. ${ }^{32}$

Der Lehrgestaltungsansatz des „Marktplatzes“ ist von umfangreichen Reformen der Ausbildungsvorschriften unabhängig. Die Reform der Juristenausbildung „von unten “ im Wege der Umsetzung innovativer Lehre direkt im Hörsaal oder Seminarraum könnte sich gegenüber einer Reform „von oben“ (Schaffung von Großstrukturen, Gesetzesänderungen, etc.) langfristig als der erfolgversprechendere Weg zu einer nachhaltigen Veränderung der Lehrqualität erweisen. Stiftungen und Drittmittelgeber sollten ihre Förderstrategien entsprechend anpassen, damit Gelder auch dort ankommen, wo sie direkt den Studierenden zugutekommen: im Hörsaal, bei den einzelnen Lehrenden. Als ausgesprochen positives Beispiel kann die Initiative des Stifterverbandes für die deutsche Wissenschaft, der Joachim-Herz-Stiftung und der Baden-Württemberg-Stiftung im Rahmen des Programms „Fellowships für Innovationen in der Hochschullehre“ gelten. ${ }^{33}$ Für eine nachhaltige Verankerung innovativer Lehre im Lehralltag der Fakultäten sollte möglichst rasch eine Institutionalisierung der Lehrförderung in einer „Deutschen Lehrgemeinschaft“ erfolgen, um ein Einzelantragsverfahren dauerhaft zu etablieren.

Poster lassen sich auch für Wettbewerbe nutzen und könnten sogar den Raum einer Fakultät insgesamt gestalten. Große Methoden wie Zukunftswerkstätten, open space oder Markt der Möglichkeiten lassen sich bei wissenschaftlichen Projekttagen der gesamten Fakultät zum Einsatz bringen. Sie eigenen sich damit auch zur Organisationsentwicklung. ${ }^{34}$ Der Vorteil, den ein Hörsaal bietet, ist seine Größe. Diesen Raum im Wortsinne als Lernraum zu benutzen, ist ein bislang wenig erprobtes Konzept, um große Lehrveranstaltungen punktuell lebhafter, abwechslungsreicher und vor allem auch forschungsnah zu gestalten. Die Nutzung des Hörsaals als Lernraum schafft neue Möglichkeiten der Begegnung und des Austausches. Nicht zuletzt hat der „Marktplatz Rechtsphilosophie“ mit den Mitteln der Raumgestaltung forschungsnahes Lernen auf neue Weise sichtbar gemacht.

32 Für einen integrativen Ansatz Schaeper/Briedis, Kompetenzen von Hochschulabsolventinnen und Hochschulabsolventen, berufliche Anforderungen und Folgerungen für die Hochschulreform, http:// www.his.de/pdf/pub_kia/kia200406.pdf (27.2.2013) S. 5 der Einleitung.

33 Siehe Stifterverband für die deutsche Wissenschaft, Fellowships für Innovationen in der Hochschullehre, http://www.stifterverband.info/wissenschaft_und_hochschule/lehre/fellowships/ (27.2.2013).

34 Siehe dazu zum Beispiel die Ausstellung auf dem Projekttag „LehrReich“ an der Fakultät für Rechtswissenschaft der Universität Hamburg, Fakultät für Rechtswissenschaft der Universität Hamburg, LehrReich - Ausstellung, http://www.jura.uni-hamburg.de/lehrreich/ausstellung/ (27.2..2013). Zum Ansatz der institutionellen Qualitätsentwicklung über die Hochschuldidaktik, Lüthje, Von der Hochschuldidaktik zur Qualitätsentwicklung, in: Merkt/Mayrberger, Die Qualität akademischer Lehre, 2007, S. 15-24. 


\section{Die GmbH nach der MoMiG-Reform}

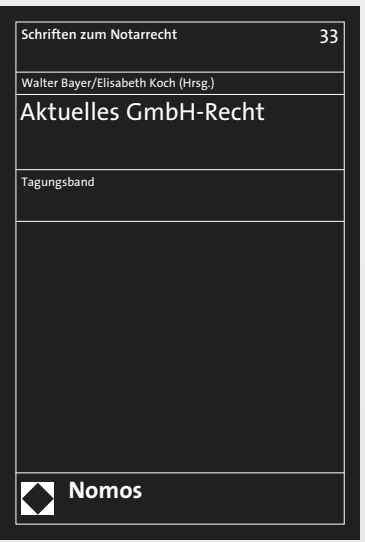

\section{Aktuelles GmbH-Recht}

Tagungsband

Herausgegeben von Prof. Dr. Walter Bayer und Prof. Dr. Elisabeth Koch

2013, 177 S., brosch., 54,-€

ISBN 978-3-8487-0084-4

(Schriften zum Notarrecht, Bd. 33)

www.nomos-shop.de/20168

Der Tagungsband dokumentiert die auf dem 7. Symposium des Instituts für Notarrecht gehaltenen Vorträge zum aktuellen $\mathrm{GmbH}$ Recht. An vielen Stellen des $\mathrm{GmbH}$-Rechts besteht auch nach der MoMiG-Reform rechtspolitischer Handlungsbedarf. Ziel ist es, dem Leser aktuelle Problemfelder aufzuzeigen und rechtspolitische Lösungsvorschläge zu unterbreiten.

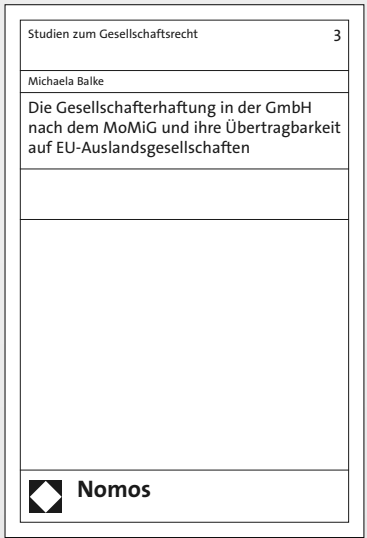

Die Gesellschafterhaftung in der $\mathrm{GmbH}$ nach dem MoMiG und ihre Übertragbarkeit auf EU-Auslandsgesellschaften

Von RAin Dr. Michaela Balke

2013, 414 S., brosch., 99,-€

ISBN 978-3-8487-0092-9

(Studien zum Gesellschaftsrecht, Bd. 3) www.nomos-shop.de/20235

Die Studie bereitet umfassend die veränderte Rechtslage auf, die sich aus dem MoMiG für die Gesellschafterhaftung (Kapitalaufbringung, Kapitalerhaltung, Recht der Gesellschafterdarlehen und Existenzvernichtungshaftung) in der deutschen $\mathrm{GmbH}$ ergibt und untersucht darüber hinaus die Frage der Anwendbarkeit dieser Haftungsinstitute auf EU-Auslandsgesellschaften. 


\section{Schriften zur Europäischen Integration und Internationalen Wirtschaftsordnung}

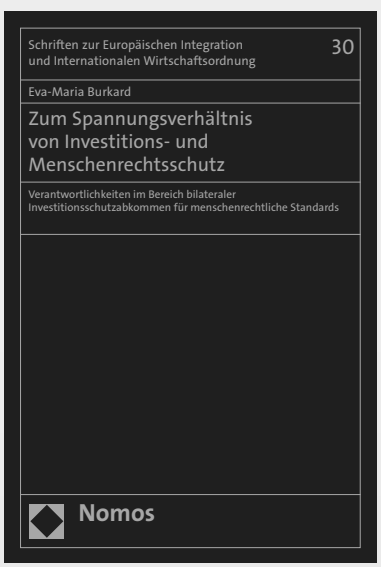

Zum Spannungsverhältnis von Investitions- und Menschenrechtsschutz

Verantwortlichkeiten im Bereich bilateraler Investitionsschutzabkommen für menschenrechtliche Standards

Von RAin Dr. Eva-Maria Burkard

2013, Band 30, 330 S., brosch., 84,- $€$

ISBN 978-3-8487-0179-7

wWw.nomos-shop.de/20356

Vor dem Hintergrund von investitionsschutzund menschenrechtlichen Verpflichtungen stellt die Arbeit exemplarisch Konflikte vor Investitionsschiedsgerichten dar und beleuchtet rechtliche Lösungsansätze. Sodann werden die Verantwortlichkeiten der beteiligten Akteure unter Berücksichtigung der Arbeit des UN-Sonderbeauftragten für Wirtschaft und Menschenrechte untersucht.

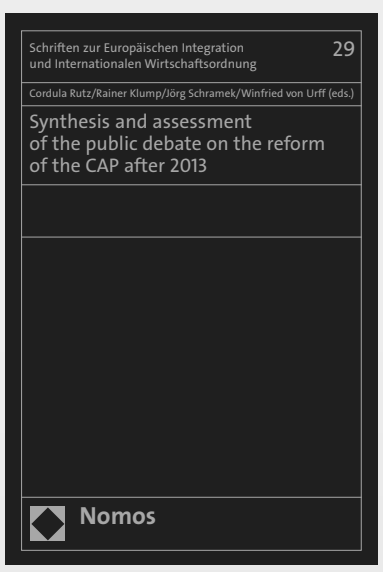

Synthesis and assessment of the public debate on the reform of the CAP after 2013

Herausgegeben von Cordula Rutz,

Prof. Dr. Rainer Klump, Jörg Schramek und Prof. Dr. Winfried von Urff

2013, Band 29, 98 S., brosch., 28,-€

ISBN 978-3-8487-0172-8

www.nomos-shop.de/20335

Diese Veröffentlichung bietet eine vergleichende Analyse der öffentlichen Debatte zur Reform der Gemeinsamen Agrarpolitik nach 2013 in zehn EU Mitgliedsstaaten. Hierzu werden die Stellungnahmen von relevanten Akteuren, wie Regierungen, Wissenschaftlern, Landwirtschafts- und Umweltverbänden, zu den Reformvorschlägen der Europäischen Kommission ausgewertet. 


\section{„Erbrecht“ Internationaler Organisationen}

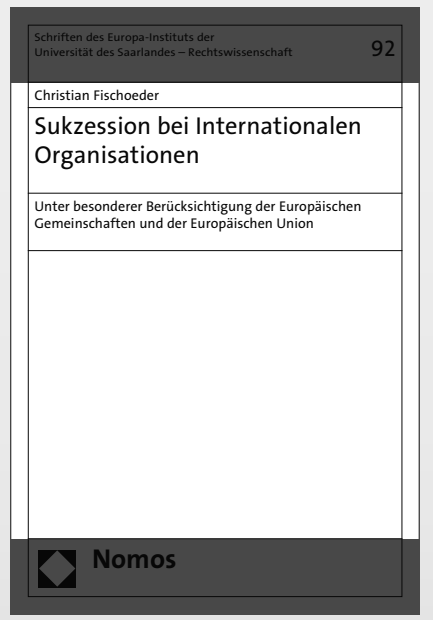

Der Rechtsbereich der Nachfolge bei Internationalen Organisationen hat bislang wenig Aufmerksamkeit erhalten - obwohl es an Beispielen nicht mangelt. Die Studie will das „Erbrecht“ der Internationalen Organisationen herausarbeiten. Hierbei werden die Sukzessionen bei der Europäischen Gemeinschaft für Kohle und Stahl, der Europäischen Gemeinschaft und der Europäischen Union genauer unter die Lupe genommen.

Die Arbeit verfolgt eine induktive Methode: Zunächst werden die bisher erfolgten Sukzes-

\section{Sukzession bei Internationalen Organisationen}

Unter besonderer Berücksichtigung der Europäischen Gemeinschaften und der Europäischen Union Von Dr. Christian Fischoeder, LL.M.

2013, 202 S., brosch., 49,- $€$

ISBN 978-3-8329-8009-2

(Schriften des Europa-Instituts der Universität des Saarlandes - Rechtswissenschaft, Bd. 92) www.nomos-shop.de/20020 sionen analysiert, um gemeinsame Vorgehensweisen zu finden und um auf möglicherweise vorhandenes Völkergewohnheitsrecht zu schließen. Die Mitgliedsstaaten Internationaler Organisationen sind bei Nachfolgeregelungen sehr um Kontinuität bemüht: Selten fällt die Aufgabe einer Organisation völlig weg; vielmehr ist diese dann von einer anderen zu bewältigen. Insoweit ist Kontinuität in den internationalen Beziehungen ein Leitmotiv der Untersuchung. 